\title{
A search for very high energy $\gamma$-ray emission from the starburst galaxy NGC 253 with HESS
}

\author{
F. Aharonian ${ }^{1}$, A. G. Akhperjanian ${ }^{2}$, A. R. Bazer-Bachi ${ }^{3}$, M. Beilicke $^{4}$, W. Benbow ${ }^{1}$, D. Berge $^{1}$, \\ K. Bernlöhr ${ }^{1,5}$, C. Boisson ${ }^{6}$, O. Bolz ${ }^{1}$, V. Borrel ${ }^{3}$, I. Braun ${ }^{1}$, F. Breitling ${ }^{5}$, A. M. Brown ${ }^{7}$, P. M. Chadwick ${ }^{7}$, \\ L.-M. Chounet ${ }^{8}$, R. Cornils ${ }^{4}$, L. Costamante ${ }^{1,20}$, B. Degrange ${ }^{8}$, H. J. Dickinson ${ }^{7}$, A. Djannati-Ataï ${ }^{9}$, \\ L. O'C. Drury ${ }^{10}$, G. Dubus ${ }^{8}$, D. Emmanoulopoulos ${ }^{11}$, P. Espigat ${ }^{9}$, F. Feinstein ${ }^{12}$, G. Fontaine ${ }^{8}$, Y. Fuchs ${ }^{13}$, S. Funk ${ }^{1}$, \\ Y. A. Gallant ${ }^{12}$, B. Giebels ${ }^{8}$, S. Gillessen ${ }^{1}$, J. F. Glicenstein ${ }^{14}$, P. Goret ${ }^{14}$, C. Hadjichristidis ${ }^{7}$, M. Hauser ${ }^{11}$, \\ G. Heinzelmann ${ }^{4}$, G. Henri ${ }^{13}$, G. Hermann ${ }^{1}$, J. A. Hinton ${ }^{1}$, W. Hofmann ${ }^{1}$, M. Holleran ${ }^{15}$, D. Horns ${ }^{1}$, \\ A. Jacholkowska ${ }^{12}$, O. C. de Jager ${ }^{15}$, B. Khélifi ${ }^{1}$, Nu. Komin ${ }^{5}$, A. Konopelko ${ }^{1,5}$, I. J. Latham ${ }^{7}$, R. Le Gallou ${ }^{7}$, \\ A. Lemière ${ }^{9}$, M. Lemoine-Goumard ${ }^{8}$, N. Leroy ${ }^{8}$, T. Lohse ${ }^{5}$, J. M. Martin ${ }^{6}$, O. Martineau-Huynh ${ }^{16}$, A. Marcowith ${ }^{3}$, \\ C. Masterson ${ }^{1,20}$, T. J. L. McComb ${ }^{7}$, M. de Naurois ${ }^{16}$, S. J. Nolan 7 , A. Noutsos ${ }^{7}$, K. J. Orford ${ }^{7}$, J. L. Osborne ${ }^{7}$, \\ M. Ouchrif ${ }^{16,20}$, M. Panter ${ }^{1}$, G. Pelletier ${ }^{13}$, S. Pita ${ }^{9}$, G. Pühlhofer ${ }^{1,11}$, M. Punch ${ }^{9}$, B. C. Raubenheimer ${ }^{15}$, M. Raue ${ }^{4}$, \\ J. Raux ${ }^{16}$, S. M. Rayner ${ }^{7}$, A. Reimer ${ }^{17}$, O. Reimer ${ }^{17}$, J. Ripken ${ }^{4}$, L. Rob ${ }^{18}$, L. Rolland ${ }^{16}$, G. Rowell ${ }^{1}$, V. Sahakian ${ }^{2}$, \\ L. Saugé ${ }^{13}$, S. Schlenker ${ }^{5}$, R. Schlickeiser ${ }^{17}$, C. Schuster ${ }^{17}$, U. Schwanke ${ }^{5}$, M. Siewert ${ }^{17}$, H. Sol ${ }^{6}$, D. Spangler ${ }^{7}$, \\ R. Steenkamp ${ }^{19}$, C. Stegmann ${ }^{5}$, J.-P. Tavernet ${ }^{16}$, R. Terrier ${ }^{9}$, C. G. Théoret ${ }^{9}$, M. Tluczykont ${ }^{8,20}$, G. Vasileiadis ${ }^{12}$, \\ C. Venter ${ }^{15}$, P. Vincent ${ }^{16}$, H. J. Völk ${ }^{1}$, and S. J. Wagner ${ }^{11}$ \\ (Affiliations are given below the references)
}

Received 26 May 2005 / Accepted 12 July 2005

\section{ABSTRACT}

We present the result of $28 \mathrm{~h}$ of observations of the nearby starburst galaxy NGC 253 with the HESS detector in 2003 . We find no evidence for very high energy $\gamma$-ray emission from this object. Gamma-ray emission above $400 \mathrm{GeV}$ from NGC 253 had been reported by the CANGAROO collaboration in 2002. From the HESS data we derive upper limits on the flux above $300 \mathrm{GeV}$ of $1.9 \times 10^{-12}$ photons $\mathrm{cm}^{-2} \mathrm{~s}^{-1}$ for a point-like source and $6.3 \times 10^{-12}$ photons $\mathrm{cm}^{-2} \mathrm{~s}^{-1}$ for a source of radius $0.5^{\circ}$ as reported by CANGAROO, both at a confidence level of $99 \%$. These upper limits are inconsistent with the spectrum reported by CANGAROO. The expected very high energy $\gamma$-ray emission from this object is discussed in the framework of a galactic wind propagation model.

Key words. gamma-rays: observations - galaxies: starburst - galaxies: individual objects: NGC 253

\section{Introduction}

Starburst galaxies, such as the closest objects of this type, NGC 253 in the Southern and M 82 in the Northern Hemisphere, are known to exhibit enhanced and strongly localised supernova explosion rates in so called "starburst regions". In such regions gas and photon densities are very high compared to the situation in normal star forming galaxies like the Milky Way. Given the paradigm of cosmic-ray (CR) acceleration in supernova remnants (SNR), substantially greater CR energy densities $E_{\mathrm{c}} \geq 100 \times E_{\mathrm{c}}^{\mathrm{gal}}$ (where $E_{\mathrm{c}}^{\mathrm{gal}} \sim 1 \mathrm{eV} \mathrm{cm}^{-3}$ is the value in our galaxy) are expected in such regions, with corresponding fluxes for $\pi^{0}$-decay $\gamma$-rays alone in the range of $10^{-9} \leq F_{\gamma}(>300 \mathrm{MeV}) \leq 10^{-8}$ photons $\mathrm{cm}^{-2} \mathrm{~s}^{-1}$ for an object such as M 82 (Völk et al. 1989). Later estimates for M 82 (Akyüz et al. 1991; Völk et al. 1996) are approximately encompassed by this range, which translates into a $\gamma$-ray flux of $1.5 \times 10^{-13} \leq F_{\gamma}(>1 \mathrm{TeV}) \times\left(E_{\gamma} / 1 \mathrm{TeV}\right)^{1.1} \leq 1.5 \times 10^{-12}$ photons $\mathrm{cm}^{-2} \mathrm{~s}^{-1}$ at energies $E_{\gamma} \gg 300 \mathrm{MeV}$ assuming a differential proton energy spectrum $\propto E_{\mathrm{p}}^{-2.1}$.
EGRET observations of NGC 253 (Sreekumar et al. 1994), the nearest spiral galaxy outside the local group (at distance $d \approx 2.6 \mathrm{Mpc}$ ), resulted in an upper limit on the integral flux above $100 \mathrm{MeV}$ of $1.0 \times 10^{-7}$ photons $\mathrm{cm}^{-2} \mathrm{~s}^{-1}$. A reanalysis of these data by Paglione et al. (1996) yielded a consistent limit of $8 \times 10^{-8}$ photons $\mathrm{cm}^{-2} \mathrm{~s}^{-1}$. More complete EGRET data led Blom et al. (1999) to revise this upper limit down to $3.4 \times$ $10^{-8}$ photons $\mathrm{cm}^{-2} \mathrm{~s}^{-1}$; for M 82 they derive an upper limit of $4.4 \times 10^{-8}$ photons $\mathrm{cm}^{-2} \mathrm{~s}^{-1}$. Similar upper limits for both NGC 253 and M 82 were obtained in a recent reanalysis of the EGRET data by Cillis et al. (2005). NGC 253 was detected in the hard X-ray band using the OSSE instrument (Bhattacharya et al. 1994). From the $4.4 \sigma$ significance detection a flux of $3 \times$ $10^{-11} \mathrm{erg} \mathrm{cm}^{-2} \mathrm{~s}^{-1}(50-165 \mathrm{keV})$ was derived.

At TeV energies, observations of both NGC 253 and M 82 were made by the HEGRA collaboration, but neither object was detected (Götting 2005). For NGC 253 a flux upper limit of $F_{\gamma}(>5.2 \mathrm{TeV})<1.3 \times 10^{-13}$ photons $\mathrm{cm}^{-2} \mathrm{~s}^{-1}$ at $99 \%$ confidence was derived from 32.5 hours of live time. For M 82 
the corresponding limit was $F_{\gamma}(>2.1 \mathrm{TeV})<2.7 \times 10^{-13}$ photons $\mathrm{cm}^{-2} \mathrm{~s}^{-1}$ from 43.9 hours of live time. The report of very high energy (VHE, used here as $0.1 \mathrm{TeV}-10 \mathrm{TeV}$ ) $\gamma$-ray emission from NGC 253 by the CANGAROO collaboration (Itoh et al. 2002) marked the first claim of $\gamma$-ray emission from a starburst galaxy. The differential energy spectrum (Itoh et al. $2003 \mathrm{~b})$ can be described by: $\mathrm{d} F / \mathrm{d} E=(2.85 \pm 0.71) \times 10^{-12} \times$ $(E / 1 \mathrm{TeV})^{-3.85 \pm 0.46} \mathrm{~cm}^{-2} \mathrm{~s}^{-1} \mathrm{TeV}^{-1}$, with a corresponding flux above $400 \mathrm{GeV}$ of $\approx 1.4 \times 10^{-11} \mathrm{~cm}^{-2} \mathrm{~s}^{-1}$, or $\approx 15 \%$ of the flux of the Crab Nebula above the same threshold. The emission reported by CANGAROO is extended with an rms angular size estimated as $0.3-0.6^{\circ}$, somewhat larger than the optical size of the galaxy $\left(28^{\prime} \times 7^{\prime}\right)$.

Further evidence for particle acceleration in NGC 253, at lower photon energies, exists mainly in the form of radio data. Radio synchrotron observations show an extended, quasi-spherical halo, rising to at least $9 \mathrm{kpc}$ above the disk (Carilli et al. 1992), with a morphology that suggests a gas outflow from the disk, carrying with it magnetic fields and radiating electrons. Such an outflow should be characteristic for starburst galaxies. It has been studied in detail in thermal soft X-rays (e.g. Dahlem et al. 1998, Strickland et al. 2000, $2002 a, b)$ and interpreted in terms of "superwinds". The observed X-ray emission shows a good correlation with optical $\mathrm{H}_{\alpha}$ line and radio continuum observations over the same region. From general considerations of particle acceleration, it seems likely that accelerated nuclear particles are also present and contribute to driving the wind. The CANGAROO detection has been interpreted as inverse Compton emission, implying the existence of an extended halo of multi-TeV CR electrons around NGC 253 (Itoh et al. 2003). A cold dark matter (CDM) annihilation interpretation was also discussed (Enomoto et al. 2003).

The detection of NGC 253 by CANGAROO, along with the theoretical expectation of very high energy $\gamma$-rays from starburst galaxies, motivated the observation of this object with the High Energy Stereoscopic System (HESS ). HESS is an array of four $13 \mathrm{~m}$ diameter telescopes, each equipped with 960 pixel ( $5^{\circ}$ field-of-view) cameras (Hinton 2004). Commissioning of the array in Namibia (at $1800 \mathrm{~m}$ above sea level) began in 2002 and the array was completed in December 2003. The optical system of the HESS telescopes is described in detail by Bernlöhr et al. (2003) and Cornils et al. (2003), the trigger system by Funk et al. (2004) and the camera electronics by Vincent et al. (2003). Observations of NGC 253, made during 2003 with HESS, are presented here.

\section{HESS observations}

Observations of NGC 253 were made during the construction of the HESS array. In August and September 2003, $30 \mathrm{~h}$ of data were taken with two telescopes in operation. In October 2003 a further $8 \mathrm{~h}$ of 3-telescope data were taken. After run quality selection (primarily to discard data compromised by unstable weather conditions) and dead time correction, datasets of $24.6 \mathrm{~h}$ (August/September) and $3.4 \mathrm{~h}$ (October) remain. The mean zenith angle of observations was $14^{\circ}$. Only events where at least two telescopes provided shower images were used in this analysis to enable stereoscopic reconstruction. Data were taken in wobble mode, with the target source offset by $0.5^{\circ}$ from the tracking position of the telescopes. The energy threshold for this dataset is $190 \mathrm{GeV}$. Two independent analysis methods have been applied to search for $\gamma$-ray emission in this dataset.

\subsection{Analysis method 1}

The standard calibration and analysis used for HESS is described in detail in (Aharonian et al. 2005; Aharonian et al. 2004) and is referred to here as Analysis 1. Following calibration, tail-cuts image cleaning and image moment analysis (Hillas parameter determination) were used to characterise each telescope image. Shower direction reconstruction was made based on the intersection of the major axes of the individual telescope images. The angular resolution (defined by the radius containing $68 \%$ of the reconstructed events from a pointsource) is $0.14^{\circ}$ for the two telescope data set and $0.12^{\circ}$ for three telescopes. The primary $\gamma$-ray energy is estimated (with a resolution of $\approx 15 \%$ ) using the total image size in each telescope and the reconstructed shower core position. The mean reduced scaled width and length of shower images are used to select $\gamma$-ray candidate events.

Given that the observations were taken in wobble-mode, the position $0.5^{\circ}$ from the tracking position on the opposite side of the field of view (FOV) can be used for a simultaneous background measurement. Figure 1 shows the distribution of squared angular distance $\left(\theta^{2}\right)$ of candidate $\gamma$-rays from the centre of NGC 253 for the combined 2- and 3-telescope data sets. The distributions for the on-source and background measurements are consistent; no evidence for a $\gamma$-ray signal is seen. The vertical dashed lines in this figure indicate the positions of the standard HESS $\gamma$-ray selection cut for point-like sources $\left(\theta<0.14^{\circ}\right)$ and a much wider cut $\left(\right.$ at $\left.0.5^{\circ}\right)$ at the approximate extent reported by the CANGAROO collaboration.

To enable 2-dimensional mapping of the FOV a different background model is applied. The upper panel of Fig. 2 shows the significance of a point-like excess in the FOV of NGC 253 using a ring (of $0.5^{\circ}$ radius) around each position to provide a background estimate (a correction for the radial acceptance function of the instrument is made in this case). The distribution of significance within the FOV is consistent with random fluctuations of the background.

\subsection{Analysis method 2}

An independent analysis method for the HESS data (LemoineGoumard et al. 2004a, Analysis 2) has been used to confirm this result. This analysis employs not only different reconstruction and background reduction methods but also an independent detector calibration (Aharonian et al. 2004). The reconstruction method is based on a simple 3D-modeling of an electromagnetic shower assuming rotational symmetry. For each event eight parameters are reconstructed: the direction of the shower in the reference frame of the telescope, the core position on the ground, the altitude of shower maximum, the total number of emitted Cherenkov photons, and the longitudinal 

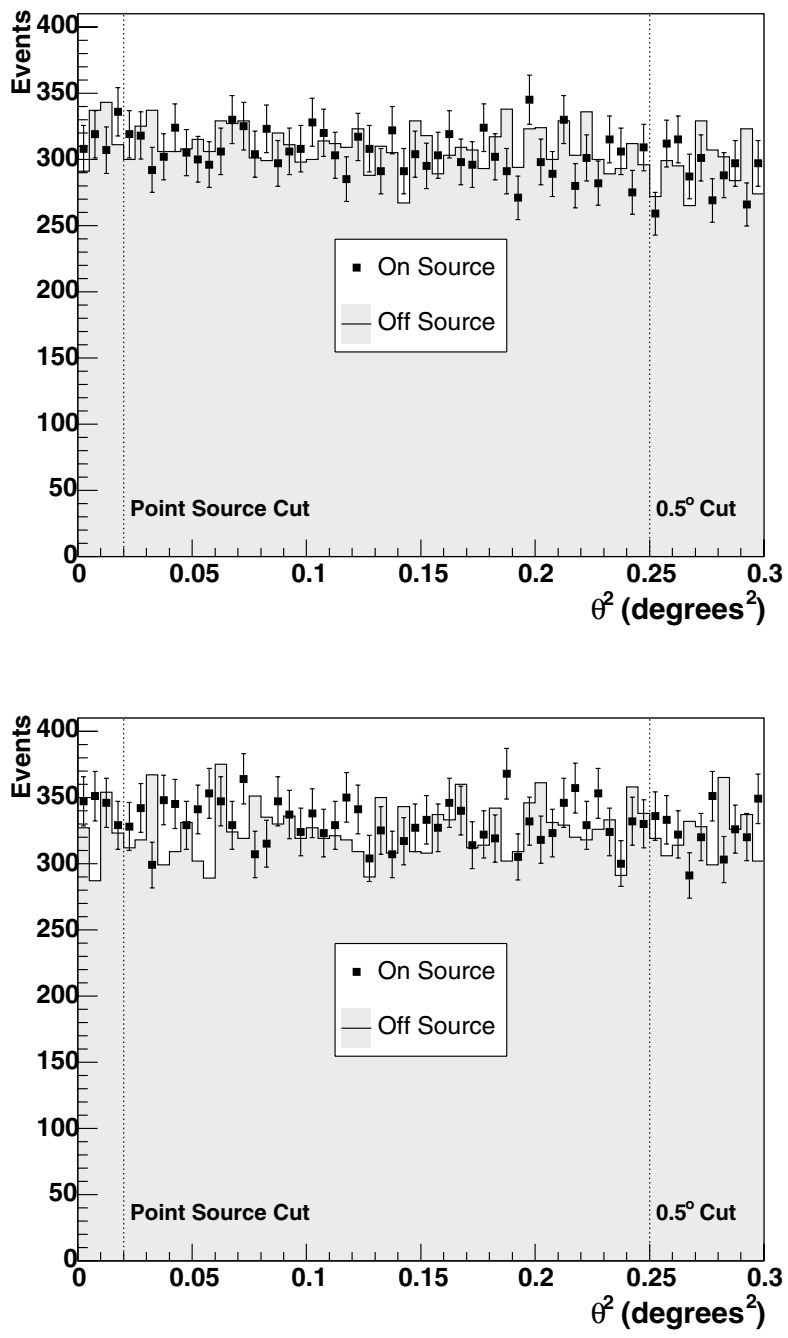

Fig. 1. Top: angular distribution of $\gamma$-like images relative to the centre of NGC 253 ("On") and relative to the background control region ("Off") for Analysis 1. Events are plotted versus the squared angular distance to give equal solid angle in each bin. Background curves (histograms) are determined relative to to points $1^{\circ}$ away from the source position. Bottom: as for upper figure but using Analysis 2 (described in Sect. 2.2).

and transverse standard deviations of the Gaussian distributions, referred to as "3D-length" and "3D-width", respectively. In this method the 3D-width is the most efficient and robust parameter to discriminate $\gamma$-ray showers from the hadronic background.

The background subtraction is performed independently in each bin of the FOV using a maximum likelihood method (Lemoine-Goumard et al. 2004b) based on the knowledge of the 3D-width distributions of $\gamma$-rays and background events, respectively. The $\gamma$-ray width distribution is determined via Monte-Carlo simulations and checked on data from known point-like sources; the distribution for background events is obtained from a FOV in the same zenith angle range with no detected sources. The dependence of these distributions on the bin position in the FOV is taken into account. The method yields two independent sky maps, one for $\gamma$-rays and the other for background events. In contrast to Analysis 1, this method makes no a priori assumption of azimuthal symmetry in the
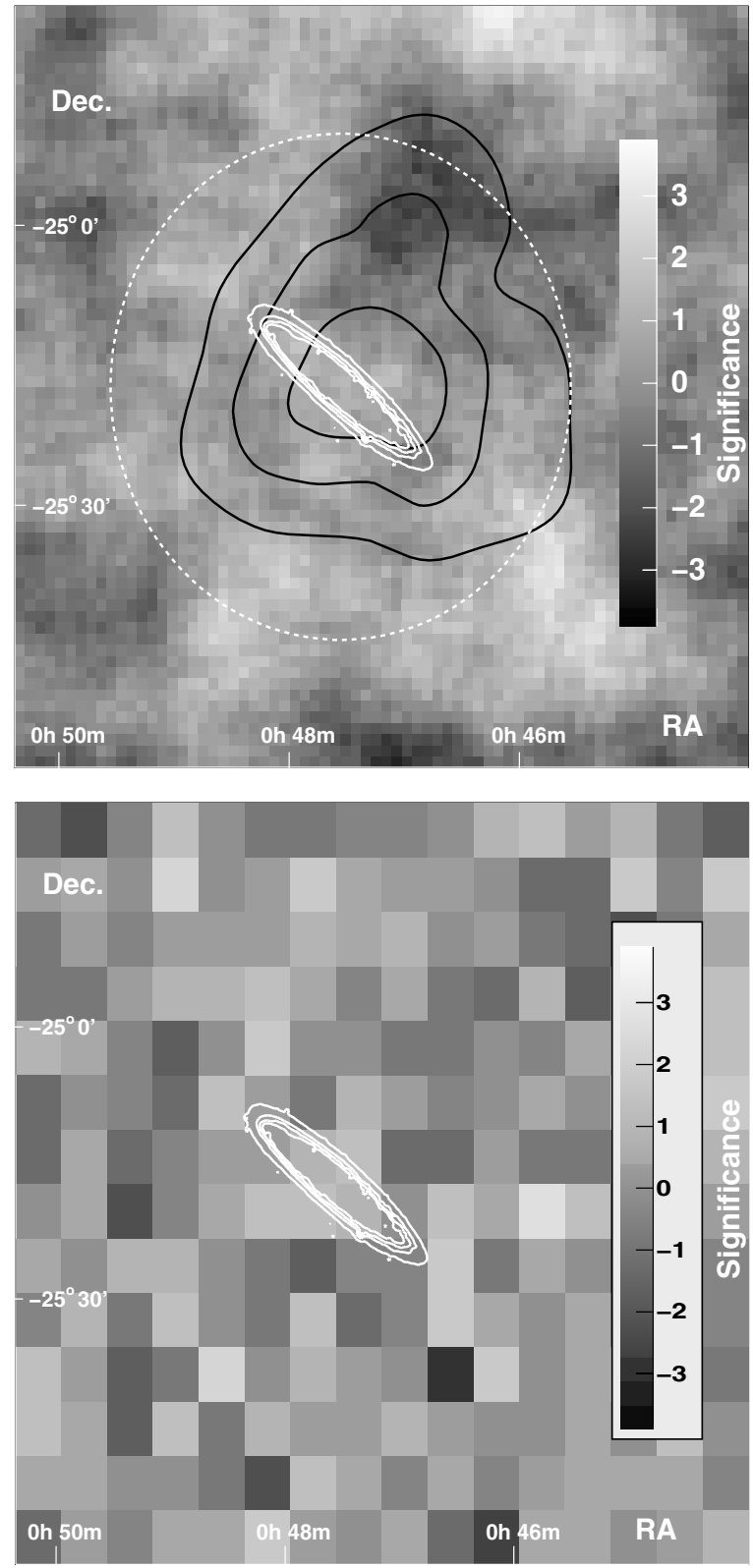

Fig. 2. Top: $\gamma$-ray point source significance map (grey-scale) derived from two telescope HESS data taken in August and September 2004 and using Analysis 1. The white contours show the optical emission from the galaxy (in a linear scale). These data are taken from the STScI Digitized Sky Survey 2004. Black contours are confidence levels (at $40 \%, 65 \%$ and $80 \%$ ) for $\mathrm{TeV} \gamma$-ray emission reported by Itoh et al. (2002). The dashed white line shows the angular cut used to derive the extended source flux limits presented here. In this approach bins are correlated within the radius of the $\theta$ cut $\left(0.14^{\circ}\right)$. Bottom: significance map derived from the same dataset using Analysis 2. In contrast to the top panel the statistical significance in each bin is independent. The bin size is matched to the angular resolution of the instrument for these data.

structure of the background. Furthermore, the $\gamma$-ray contents of different bins are statistically independent. This method was applied to the same data sets as for the analysis presented above; the significance map for $\gamma$-rays, shown in the lower panel of Fig. 2, is again compatible with the absence of a signal in the whole FOV. 


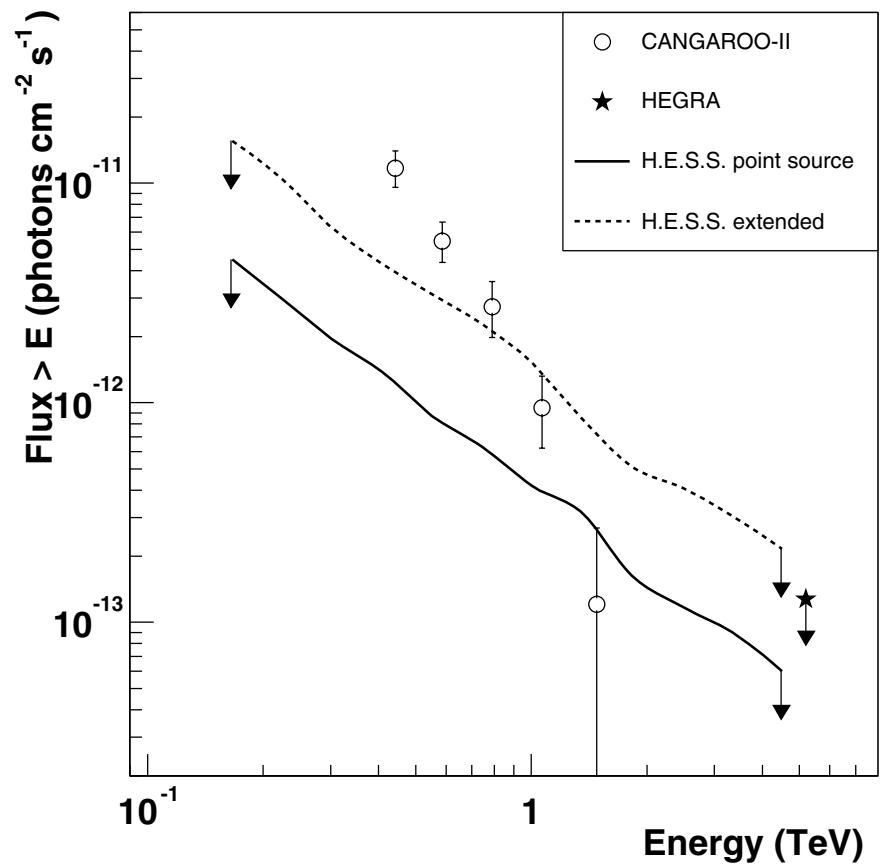

Fig. 3. Upper limits from HESS on the integral flux of $\gamma$-rays from NGC 253 (99\% confidence level) from Analysis 2. Curves for point like emission (solid lines) and for a source of $0.5^{\circ}$ radius (dashed lines) are shown. CANGAROO integral data points (derived from the differential spectrum given by Itoh et al. 2003b) and an upper limit from HEGRA (Götting 2005) are shown for comparison.

\subsection{Flux upper limits}

Figure 3 shows integral flux upper limits calculated assuming a spectrum of photon index -3.85 (as reported by CANGAROO) and following the method of Feldman \& Cousins (1998). Flux limits are shown for point-like and extended emission $\left(0.5^{\circ}\right.$ radius) for Analysis 2. Also shown are CANGAROO-II integral data points derived from the differential spectrum given by Itoh et al. (2003b). Above $300 \mathrm{GeV}$ we derive $99 \%$ confidence flux upper limits of $1.9 \times 10^{-12}$ photons $\mathrm{cm}^{-2} \mathrm{~s}^{-1}$ for a point-like source and $6.3 \times 10^{-12}$ photons $\mathrm{cm}^{-2} \mathrm{~s}^{-1}$ for a source of radius $0.5^{\circ}$. The upper limit curves derived from Analysis 1 are rather similar in shape and have slightly higher values at $300 \mathrm{GeV}: 2.2 \times 10^{-12}$ photons $\mathrm{cm}^{-2} \mathrm{~s}^{-1}$ photons $\mathrm{cm}^{-2} \mathrm{~s}^{-1}$ (point source) and $6.9 \times 10^{-12}$ photons $\mathrm{cm}^{-2} \mathrm{~s}^{-1}\left(0.5^{\circ}\right)$.

The non-detection of this object cannot be attributed to a mis-pointing of the instrument. The systematic error in the pointing error of HESS during 2003 was $<1^{\prime}$ (Gillessen et al. 2003). Moreover, the correct pointing of the instrument for this particular dataset has been independently confirmed using the currents induced by stars in the FOV in the photomultiplier-tube camera. The limit on the mis-pointing derived from this method is $<3^{\prime}$.

\section{Discussion}

Given the strong signal from NGC 253 reported by the CANGAROO collaboration, the non-detection of this object with HESS is surprising. As the source reported by Itoh et al. (2003b) is extended in nature, time-variability of the $\gamma$-ray emission cannot be invoked to solve the apparent contradiction between the HESS and the CANGAROO results. In this situation it is instructive to review the expectations for $\gamma$-ray emission from this object.

The speculative possibility of detectable $\gamma$-ray emission from Dark Matter annihilation in the halo of NGC 253 is discussed in detail in Enomoto et al. (2003). We concur with the view of these authors that such a scenario cannot produce VHE $\gamma$-rays with fluxes detectable with current instruments. The standard picture of $\gamma$-ray production via CR interactions seems more plausible given the rapidly growing knowledge about this object in other wavelength ranges, principly in terms of supernova explosion rates and the total gas mass in the starburst region. The relevant CR interactions are: i) inelastic collisions of relativistic nuclei with thermal gas nuclei producing $\pi^{0}$ mesons which decay into $\gamma$-rays ii) Inverse Compton (IC) collisions of relativistic electrons with ambient low-energy photons iii) Bremstrahlung collisions of such electrons with gas nuclei. The $\gamma$-ray flux from these processes is proportional to the density of these relativistic particles (CRs). The CR density is determined by the strength of their accelerators in the SB region, their propagation modes, and their energy losses. In particular, propagation of CRs via convection in the SB wind is a key process that is usually not considered for our own galaxy. In addition, the nature of particle diffusion in the SB is likely modified by non-linear effects induced by the CRs themselves.

A self-consistant picture for the $\gamma$-ray production is discussed below. It turns out that the strong localisation of CR production and the high gas mass of the SB region lead to a rather simple prediction for the VHE $\gamma$-ray flux, independant of the further details of the SB configuration.

\subsection{Starburst parameters for NGC 253}

The enhanced CR density and the very high gas and photon densities in the starburst (SB) regions are related to each other: the high gas densities are necessary for the high star formation rate which produces massive stars of high photon luminosity. Their subsequent supernova (SN) explosions heat the gas and generate CRs. Together the heating of the gas and the high CR density drive a mass outflow in the form of a galactic wind.

NGC 253 has a central SB region which is very bright in radio, far infrared, and $\mathrm{X}$-ray frequencies. Following Weaver et al. (2002) we assume it to be a cylinder of radius $R=150 \mathrm{pc}$ and height $H=60 \mathrm{pc}$ in the plane of the galaxy, with an outflow that is collimated by a massive molecular torus into the vertical direction, perpendicular to the disk. The outflow velocity $V_{\text {wind }}$ is not very well known, with "minimum reasonable values between 300 and $600 \mathrm{~km} \mathrm{~s}^{-1}$ " (Strickland et al. 2002a). However, values for the outflowing SN ejecta as high as $3000 \mathrm{~km} \mathrm{~s}^{-1}$ are considered possible. For the purposes of our estimates we assume here $V_{\text {wind }}=500 \mathrm{~km} \mathrm{~s}^{-1}$.

The volume of the SB region is then $V_{\mathrm{SB}} \approx 4.2 \times 10^{6} \mathrm{pc}^{3}$. The SN rate in this small nuclear volume is estimated as $v_{\mathrm{SN}}=0.03 \mathrm{yr}^{-1}$, about as high as that of our entire Galaxy (cf. Engelbracht et al. 1998), but values as high as $0.08 \mathrm{yr}^{-1}$ have 
also been estimated (van Buren \& Greenhouse 1994; Paglione et al. 1996). The rest of the galaxy is thought to have a SN rate of the same magnitude, distributed over the disk. The total (dominantly molecular) mass $M$ in the nuclear region is rather uncertain: $3 \times 10^{7} \leq M / M_{\odot} \leq 1.4 \times 10^{8}$ (Mauersberger et al. 1996, Engelbracht et al. 1998). We assume $M=6 \times 10^{7} M_{\odot}$ which corresponds to the preferred value of Engelbracht et al. (1998) and gives a density of 580 protons $\mathrm{cm}^{-3}$ in the nuclear region.

\subsection{Transport of VHE particles}

The non-thermal properties of such an SB system are quite complex, as the very extended radio synchrotron emission shows. This radio emission is intimately connected to the large expanding halo in thermal $\mathrm{X}$-rays and optical $\mathrm{H}_{\alpha}$-line photons. Nevertheless, the $\mathrm{TeV} \gamma$-ray emission can be discussed in a fairly straightforward manner. For this purpose, let us assume that particle diffusion can be calculated from the theory of the wind from our Galaxy (Ptuskin et al. 1997), scaled to the SB parameters of NGC 253. We point out that in the theory of Ptuskin et al. the diffusion coefficient in the Galactic wind is assumed to be due to Alfvén waves, excited by the particles themselves, as they stream away from their sources in the disk. The wave amplitudes are limited by nonlinear wave damping on the thermal wind plasma.

To estimate the spatial extent and magnitude of the $\gamma$ ray emission as a result of the SB, we first consider the time scales for energetic particle transport and their collisional/radiative energy losses. For protons the convection time $t_{\text {conv }}=H / V_{\text {wind }} \approx 1.2 \times 10^{5} \mathrm{yr}$, whereas the loss time in the nuclear region due to inelastic collisions with nuclei of the gas amounts to $t_{\text {loss }}=8.6 \times 10^{4} \mathrm{yr}$.

The diffusion vertically to the disk is given by the diffusion coefficient $\kappa=\left(B / B_{\text {gal }}\right)\left(\epsilon / \epsilon_{\text {gal }}\right)^{-1} \kappa_{\text {gal }}(E)$, where above a few $\mathrm{GeV} \kappa_{\text {gal }}(E) \approx 9 \times 10^{29}(E / 1 \mathrm{TeV})^{1.1} \mathrm{~cm}^{2} \mathrm{~s}^{-1}$ is consistent with the mean matter thickness traversed by the CRs in our Galaxy (and its rigidity dependance), before they are ultimately convected away into extragalactic space (Ptuskin et al. 1997) ${ }^{1}$. In this scaling relation $\epsilon \propto v_{\mathrm{SN}} /(C R$ source surface area) denotes the spatial energy flux density of CRs released into the SB region. In detail, $\epsilon=4 \pi \mathrm{c} \int_{0}^{\infty} p^{3} Q(p) \mathrm{d} p$, where $Q(p)$ is the $\mathrm{CR}$ source power density, as a function of particle momentum $p$, per unit surface area of the source region. As we shall see, in this case $t_{\text {loss }}$ is smaller than both $t_{\text {conv }}$ and $t_{\text {diff }}$ (which is defined below). This results in an upper limit for the $\gamma$-ray emission from NGC 253 for a given energy input into CRs.

To determine $\epsilon$ for NGC 253 we note that the cylindrical SB region has a radius of $150 \mathrm{pc}$, according to the above model, whereas the corresponding region of our Galaxy, the disk, has

\footnotetext{
${ }^{1}$ In our galaxy $V_{\text {wind }} \propto s$ for $s<15 \mathrm{kpc}$ where $s$ is the height above the disk, The distance $s_{\star}$ within which the energetic particles can diffusively return to the disk to produce secondaries is given by $\kappa_{\text {gal }}(E) / s_{\star}^{2}=V_{\text {wind }}\left(s_{\star}\right) / s_{\star}$ (the diffusion-convection boundary). Thus $s_{\star} \propto \kappa_{\text {gal }}^{1 / 2}(E) \propto E^{0.55}$ for our galaxy. The probability for a particle to cross the disk, and thus the mean matter thickness traversed by particles of energy $E$ is inversely proportional to $s_{\star}$.
}

a radius of $15 \mathrm{kpc}$. Therefore, assuming $v_{\mathrm{SN}}=0.03 \mathrm{yr}^{-1}$ in the SB as in the Milky Way, yields $\epsilon / \epsilon_{\mathrm{gal}}=10^{4}$. For the magnetic field $B$ in the $\mathrm{SB}$ region we chose $B \leq 270 \mu \mathrm{G}$, where $270 \mu \mathrm{G}$ corresponds to the equipartition value derived by Weaver et al. (2002) which we consider the maximum possible field in the SB region. With a Galactic field strength $B_{\text {gal }}=3 \mu \mathrm{G}$ this massive $\mathrm{CR}$ production reduces the diffusion coefficient to $\kappa \leq 9 \times 10^{-3} \kappa_{\text {gal }}$. The corresponding diffusion time $t_{\text {diff }}=H^{2} / \kappa \geq 1.3 \times 10^{5} \mathrm{yr}$ for a $1 \mathrm{TeV}$ particle also exceeds $t_{\text {loss }}$. This means that CR protons with energies up to about $1 \mathrm{TeV}$ behave calorimetrically: i.e. they lose all their energy by inelastic collisions before being able to leave the SB region. However, the increase of $\kappa$ with particle energy makes the particles with energies above several $\mathrm{TeV}$ diffusion dominated, which implies that they escape the SB region before interacting. The turnover point is at $\gamma$-ray energies of a few hundred $\mathrm{GeV}$.

For TeV electrons the loss time is estimated as follows: the enormous far infrared (FIR) SB-luminosity $L_{\mathrm{SB}}=1.1 \times 10^{10} L_{\odot}$ deduced by Engelbracht et al. (1998) implies a radiation energy density $U_{\text {rad }}^{\mathrm{SB}} \approx 500 \mathrm{eV} \mathrm{cm}^{-3}$. This leads to an inverse Compton (IC) loss time $t_{\text {loss }}^{\mathrm{IC}} \approx 180(E / 4 \mathrm{TeV})^{-1}$ yr for the electrons of energy $E \approx 4 \mathrm{TeV}$ that produce $1 \mathrm{TeV} \gamma$-rays. The IC scattering is here considered in the Thomson limit which applies for FIR target photons. Therefore, comparing with the proton emission, the SB region acts even more calorimeterically for the emission of $\mathrm{TeV} \gamma$-rays via IC scattering.

\subsection{VHE $\gamma$-ray emission}

The hadronic $\gamma$-ray emission in this scenario simply corresponds to the total amount of CR energy created. The $\gamma$-ray energy spectrum corresponds to that of the SNR sources, assumed here to be $\mathrm{d} N_{\mathrm{CR}} / \mathrm{d} E \propto E^{-2.1}$. In this picture diffusion is irrelevant below a few $\mathrm{TeV}$, and the total $\mathrm{CR}$ energy $E_{\mathrm{CR}}$ in the SB region amounts to

$E_{\mathrm{CR}} \approx 1.5 \times 10^{53} \mathrm{erg}\left[\frac{v_{\mathrm{SN}}}{0.03 \mathrm{yr}^{-1}} \frac{\Theta E_{\mathrm{SN}}}{10^{50} \mathrm{erg}} \frac{t_{\mathrm{eff}}}{5 \times 10^{4} \mathrm{yr}}\right]$,

where $t_{\text {eff }}^{-1}=t_{\text {loss }}^{-1}+t_{\text {conv }}^{-1}+t_{\text {diff }}^{-1}$, and $\Theta<1$ is the CR energy production efficiency for an average $\mathrm{SN}$ event with a mechanical energy release $E_{\mathrm{SN}}$. The CR energy density $E_{\mathrm{c}}^{253}=$ $E_{\mathrm{CR}} /$ Volume amounts to $E_{\mathrm{c}}^{253} \approx 650 \mathrm{eV} \mathrm{cm}^{-3}$. This has to be compared to the Galactic value $E_{\mathrm{c}}^{\mathrm{gal}} \sim 1 \mathrm{eV} \mathrm{cm}^{-3}$. Assuming the radiating $\mathrm{TeV}$ particles to freely penetrate the gas in and around their sources - a premise which would become more and more questionable at lower energies on account of the shorter scattering mean free path $\lambda=3 \kappa(E) / c-$ the energy spectrum of the hadronic $\gamma$-ray flux can be calculated from the following relation (cf. Völk et al. 1996):

$$
\begin{aligned}
F_{\gamma}(>E) & =1.7 \times 10^{-13} \text { photons cm }{ }^{-2} \mathrm{~s}^{-1} \\
\times & {\left[\left(\frac{E}{1 \mathrm{TeV}}\right)^{-1.1}\left(\frac{E_{\mathrm{c}}}{1 \mathrm{eV} \mathrm{cm}^{-3}}\right)\left(\frac{d}{1 \mathrm{Mpc}}\right)^{-2}\left(\frac{M}{10^{9} M_{\odot}}\right)\right] . }
\end{aligned}
$$

Substituting our preferred values for $d, M$, and $E_{\mathrm{c}}$, as calculated above, we obtain $F_{\gamma}(>E)=9.8 \times 10^{-13} \times \delta \times$ $(E / 1 \mathrm{TeV})^{-1.1}$ photons $\mathrm{cm}^{-2} \mathrm{~s}^{-1}$ up to a few hundred $\mathrm{GeV}$, and 
significantly falling off at higher $\gamma$-ray energies. The factor $\delta$ denotes the uncertainty of the assumed parameters, mainly $v_{\mathrm{SN}}$, $M$, and $\Theta$. Assuming that $\Theta \approx 0.1$ and the average $E_{\mathrm{SN}}=$ $10^{51} \mathrm{erg}$, we obtain $0.5<\delta<5$.

As long as $M \geq 6 \times 10^{7} M_{\odot}$ we have $t_{\mathrm{eff}} \approx t_{\text {loss }} \propto V_{\mathrm{SB}} / M$. Since $E_{\mathrm{c}} \propto t_{\text {loss }} / V_{\mathrm{SB}}, F_{\gamma}$ (where $\left.F_{\gamma} \propto E_{\mathrm{c}} \cdot M\right)$ then becomes independent of $M, V_{\mathrm{SB}}, t_{\mathrm{conv}}$ and $t_{\mathrm{diff}}$, and is only proportional to the energy input per SN explosion $\Theta E_{\mathrm{SN}}$ and the $\mathrm{SN}$ rate $v_{\mathrm{SN}}$, apart from the geometrical factor $d^{-2}$. This is the consequence of the calorimetric behaviour of the system.

The CR electron energy produced in the SNR sources of the SB can be assumed to amount to no more than a fraction $\sim 10^{-2}$ of the energy generated in nuclear CR particles, as is the case in our galaxy. The contribution of IC scattering to the $\gamma$-ray emission in the VHE region can therefore be safely disregarded. The above hadronic flux value, with $\delta=1$, is similar to - in fact somewhat larger than - the HESS upper limit for the VHE $\gamma$-ray flux. At the most relevant energy of about $200 \mathrm{GeV}$ the predicted flux is about a factor of 3 larger than the HESS limit for an assumed point source. This demonstrates the physical relevance of the HESS upper limit measurement.

Our discussion rests on the assumption that the propagation properties of energetic particles in our own Galaxy and its halo can be scaled to the SB parameters of NGC 253. If this assumption does not hold, then some fraction of the accelerated particles will escape the galaxy before radiating, and the predicted $\gamma$-ray flux from NGC 253 will decrease relative to the estimate given here.

The emission from the rest of the galaxy may be spatially extended. However, assuming a situation roughly equal to that of the Milky Way, where the amount of matter traversed by a $\mathrm{TeV}$ particle corresponds to less than $1.5 \%$ of the hadronic interaction length, the majority of the protons will escape and will not transfer their energy to $\gamma$-rays. Therefore their total $\gamma$-ray emission is substantially smaller than that from the SB region. The IC $\gamma$-ray flux from the associated electrons is still negligible compared to the CANGAROO flux.

This scenario leads to the prediction that VHE $\gamma$-ray emission from the SB in NGC 253 will be highly localised, with an extent of less than 30 arcsec. Such emission would appear point-like for current VHE $\gamma$-ray instruments. As discussed earlier, extended emission from the galaxy as a whole is expected to be rather faint in comparison to the flux reported by the CANGAROO collaboration. In addition the expected spectrum should be rather hard as discussed earlier, again in contrast to the reported CANGAROO spectrum.

\section{Conclusions}

The recently completed HESS instrument is currently the most sensitive detector of $\gamma$-rays at VHE energies. The non-detection of NGC 253 by HESS is surprising given the rather high flux previously claimed by Itoh et al. (2002). From theoretical considerations of $\gamma$-ray production in starburst galaxies it appears that the HESS limit is close to the expected flux from the NGC 253 starburst, already excluding extreme parameter values $(\delta>1)$. However, a diffusive transport away from the starburst region that is faster than assumed here cannot be excluded experimentally. The possible $\gamma$-ray flux would then be correspondingly reduced.

It also seems likely that VHE $\gamma$-ray emission from NGC 253 should be rather localised (indeed point-like for current detectors), given the characteristics of the starburst measured at other wavelengths. A hard $\gamma$-ray photon index $(\approx 2)$ would also be expected.

Despite this non-detection, NGC 253 remains one of the most promising starburst galaxies for detection in very high energy $\gamma$-rays. The likely calorimetric character of this system makes deeper observations an interesting proposition.

Acknowledgements. The support of the Namibian authorities and of the University of Namibia in facilitating the construction and operation of HESS is gratefully acknowledged, as is the support by the German Ministry for Education and Research (BMBF), the Max Planck Society, the French Ministry for Research, the CNRS-IN2P3 and the Astroparticle Interdisciplinary Programme of the CNRS, the UK Particle Physics and Astronomy Research Council (PPARC), the IPNP of the Charles University, the South African Department of Science and Technology and National Research Foundation, and by the University of Namibia. We appreciate the excellent work of the technical support staff in Berlin, Durham, Hamburg, Heidelberg, Palaiseau, Paris, Saclay, and in Namibia in the construction and operation of the equipment. We thank V. N. Zirakashvili for discussions regarding the transport properties of starburst galaxies.

\section{References}

Aharonian, F., Akhperjanian, A. G., Aye, K.-M., et al. (HESS Collaboration) 2004, Astropart. Phys., 22, 109

Aharonian, F., Akhperjanian, A. G., Aye, K.-M., et al. (HESS Collaboration) 2005, A\&A, 430, 865

Akyüz, A., Broulliet, N., \& Özel, M. E. 1991, A\&A, 248, 419

Bernlöhr, K., Carrol, O., \& Cornils, R., et al. 2003, APh., 20, 111

Bhattacharya, D., The, T.-S., Kurfess, J. D., et al. 1994, ApJ, 437, 173

Blom, J. J., Paglione, T. A. D., \& Carraninana, A. 1999, ApJ, 516, 744

Carilli, C. L., Holdaway, M. A., Ho, P. T. P., et al. 1992, ApJ, 399, L59

Cillis, A. N., Torres, D. F., \& Reimer, O. 2005, ApJ, 621, 139

Cornils, R., Gillessen, S., Jung, I., et al. 2003, APh., 20, 129

Dahlem, M., Weaver, K. A., \& Heckman, T. M. 1998, ApJS, 118, 401

Engelbracht, C. W., Rieke, M. J., Rieke, G. H., et al. 1998, ApJ, 505, 639

Enomoto, R., Yoshida, T., Yanagita, S., Itoh, C., et al. 2003, ApJ, 596, 216

Feldman, G. J., \& Cousins, R. D. 1998, Phys. Rev. D, 57, 7

Funk, S., Hermann, G., Hinton, J., et al. 2004, Astropart. Phys. 22, 285

Gillessen, S., for the HESS collaboration, 2003, Proc. 28th ICRC, Tsukuba (Tokyo: Univ. Academy Press), 2899

Götting, N. 2005, Ph.D. Thesis, in preparation

Hinton, J. A. (HESS Collaboration), 2004, New Astron. Rev., 48, 331

Itoh, C., Enomoto, R., Yanagita, S., et al. 2002, A\&A, 396, L1

Itoh, C., Enomoto, R., Yanagita S., Yoshida, T., \& Tsuru, T. G. 2003, ApJ, 584, L65

Itoh, C., Enomoto, R., Yanagita, S., et al. 2003b, A\&A, 402, 443

Lemoine-Goumard, M., et al. 2004a, Proc. HDGS, Heidelberg, 703

Lemoine-Goumard, M., et al. 2004b, Proc. HDGS, Heidelberg, 697

Mauersberger, R., Henkel, C., Wielebinski, R., et al. 1996, A\&A, 305, 421

Paglione, T. A. D., Marscher, A. P., Jackson, J. M., et al. 1996, ApJ, 460,295 
Ptuskin, V. S., Völk, H. J., Zirakashvili, V. N., \& Breitschwerdt, D. 1997, A\&A, 321, 434

Sreekumar, P., Bertsch, D. L., Dingus, B. L., et al. 1994, ApJ., 426, 105

Strickland, D. K., Heckman, T. M., Weaver, K. A., et al. 2000, ApJ, 120, 2965

Strickland, D. K., Heckman, T. M., Weaver, K. A., et al. 2002a, ApJ, 568,689

Strickland, D. K., Heckman, T. M., Colbert, E. J. M., et al. 2002b, ApJ, 606, 829

STScI Digitized Sky Survey, http://stdatu.stsci.edu/

van Buren, D., Greenhouse, M. A. 1994, ApJ, 431, 640

Vincent P., Denance J.-P., Huppert J.-F., et al. 2003, Proc. 28th ICRC, Tsukuba, (Tokyo: Univ. Academy Press), 2887

Völk, H. J., Klein, U., \& Wielebinski, R. 1989, Astron. Astrophys., 213, L12

Völk, H. J., Aharonian, F. A., Breitschwerdt, D. 1996, Space Sci. Rev., 75,279

Weaver, K. A., Heckman, T. M., Strickland, D. K., \& Dahlem, M. 2002, ApJ, 576, L 19

1 Max-Planck-Institut für Kernphysik, Heidelberg, Germany e-mail: Jim.Hinton@mpi-hd.mpg.de

2 Yerevan Physics Institute, Armenia

3 Centre d'Étude Spatiale des Rayonnements, CNRS/UPS, Toulouse, France
${ }^{4}$ Universität Hamburg, Institut für Experimentalphysik, Germany

5 Institut für Physik, Humboldt-Universität zu Berlin, Germany

${ }^{6}$ LUTH, UMR 8102 du CNRS, Observatoire de Paris, Section de Meudon, France

7 University of Durham, Department of Physics, UK

8 Laboratoire Leprince-Ringuet, IN2P3/CNRS, École Polytechnique, Palaiseau, France

9 APC, Paris, France (UMR 7164, CNRS, Université Paris VII, CEA, Observatoire de Paris)

${ }^{10}$ Dublin Institute for Advanced Studies, Ireland

11 Landessternwarte, Königstuhl, Heidelberg, Germany

12 Laboratoire de Physique Théorique et Astroparticules, IN2P3/CNRS, Université Montpellier II, France

13 Laboratoire d'Astrophysique de Grenoble, INSU/CNRS, Université Joseph Fourier, France

14 DAPNIA/DSM/CEA, CE Saclay, Gif-sur-Yvette, Cedex, France

15 Unit for Space Physics, North-West University, Potchefstroom, South Africa

${ }^{16}$ Laboratoire de Physique Nucléaire et de Hautes Énergies, IN2P3/CNRS, Universités Paris VI \& VII, Paris, France

17 Institut für Theoretische Physik, Lehrstuhl IV: Weltraum und Astrophysik, Ruhr-Universität Bochum, Germany

18 Institute of Particle and Nuclear Physics, Charles University, Prague, Czech Republic

19 University of Namibia, Windhoek, Namibia

${ }^{20}$ European Associated Laboratory for Gamma-Ray Astronomy, jointly supported by CNRS and MPG 